\title{
ON CONVERGENCE FIELDS OF NÖRLUND MEANS ${ }^{1}$
}

\author{
ALEXANDER PEYERIMHOFF
}

A Nörlund mean $N(p)$ is defined by

(1) $\quad \sigma_{n}=\frac{1}{P_{n}} \sum_{\nu=0}^{n} p_{n-\nu} s_{\nu} \quad\left(n \geqq N\right.$ with $P_{n}=\sum_{\nu=0}^{n} p_{\nu} \neq 0$ for $\left.n \geqq N\right)$.

If $\sigma_{n}=s+o(1)$ when $n \rightarrow \infty$, the sequence $\left\{s_{\nu}\right\}$ (of complex numbers) is said to be limitable $N(p)$ to the value $s$. If $\sigma_{n}=o(1)$, we shall write $s_{n} \in o(N(p))$, denoting by $o(N(p))$ the set of all the sequences limitable $N(p)$ to zero. If $\sigma_{n}=a(1)$ when $n \rightarrow \infty,,^{2}$ the sequence $\left\{s_{\nu}\right\}$ is said to be absolutely limitable $N(p)$, and we shall write $s_{n} \in a(N(p))$, denoting by $a(N(p))$ the set of all the sequences absolutely limitable $N(p)$.

A Nörlund mean is called regular if any convergent sequence $s_{n} \rightarrow s$ is transformed by (1) into a convergent sequence $\sigma_{n} \rightarrow s$. Necessary and sufficient conditions in terms of the sequence $\left\{p_{n}\right\}$ (of complex numbers) in order that (1) be regular are

$$
p_{n}=o\left(P_{n}\right) \quad(n \rightarrow \infty),
$$

and

$$
\sum_{n=0}^{n}\left|p_{n}\right|=O\left(P_{n}\right) \quad(n \rightarrow \infty) .
$$

Similarly a Nörlund mean is called absolutely regular if any sequence $\left\{s_{\nu}\right\}$ with $s_{n} \rightarrow s$ and $s_{n}=a(1)$ is transformed by (1) into a sequence $\left\{\sigma_{n}\right\}$ with $\sigma_{n} \rightarrow s$ and $\sigma_{n}=a(1)$, and $N(p)$ is absolutely regular if and only if the conditions (2) and

$$
\sum_{n \geqq \operatorname{Max}(k, N+1)}\left|\frac{P_{n-k}}{P_{n}}-\frac{P_{n-1-k}}{P_{n-1}}\right| \leqq K,
$$

$K$ independent of $k(k=1,2, \cdots)$,

are valid. ${ }^{3}$

Received by the editors May 13, 1955.

1 This research was supported by the United States Air Force, through the Office of Scientific Research of the Air Research and Development Command.

2 Similarly to the symbols $O$ and $O$ the condition $a_{n}=a\left(b_{n}\right)$ means that there is a sequence $\left\{\alpha_{n}\right\}$ with $a_{n}=\alpha_{n} b_{n}\left(n \geqq n_{0}\right)$ and $\left\{\alpha_{n}\right\}$ absolutely convergent, i.e. $\sum \mid \alpha_{n}$ $-\alpha_{n+1} \mid<\infty$.

3 Cf. Mears [4] and Knopp-Lorentz [2]. 
With any regular (respectively absolutely regular) Nörlund mean $N(p)$ there is associated a function $p(z)=\sum_{0}^{\infty} p_{n} z^{n}$ regular for $|z|<1 .^{4}$

Consider now two Nörlund means $N(p)$ and $N(r)$ where $N(p)$ is regular while $r(z)=\sum_{0}^{\infty} r_{n} z^{n}$ is regular for $|z| \leqq 1$ with $r(0) \neq 0$, $r(1) \neq 0$. Then $q(z)=r(z) p(z)$ belongs to a regular Nörlund mean $N(q)$. In this paper we shall ask for the relation between the convergence fields of the Nörlund means $N(p)$ and $N(r)$ on the one hand and the convergence field of the Nörlund mean $N(q)$ on the other hand. We shall show that there holds a certain additive relation (the following results are special cases of Theorem 1 ).

If $r(z) \neq 0$ for $|z|=1$ and $p(z) \neq 0$ for $|z|<1$, then $s_{n} \in o(N(q))$ if and only if $s_{n}=u_{n}+v_{n}$ where $u_{n} \in o(N(r))$ and $v_{n} \in o(N(p))$.

If we assume that $N(p)$ is absolutely regular, then the Nörlund mean $N(q)$ is absolutely regular. There holds a similar additive relation if $o$ is replaced by $a$.

If $r(z) \neq 0$ for $|z|=1$ and $p(z) \neq 0$ for $|z|<1$, then $s_{n} \in a(N(q))$ if and only if $s_{n}=u_{n}+v_{n}$ where $u_{n} \in a(N(r))$ and $v_{n} \in a(N(p))$.

In the case $p(z)=1$ we are able to give explicitly all the sequences of $o(N(q))$ respectively $a(N(q))$. Suppose that $\left\{r_{n}\right\}$ satisfies the conditions of the first theorem above. Let $\alpha_{i}(i=1,2, \cdots, \kappa)$ be the zeros of $r(z)$ for $|z|<1, \alpha_{i}$ having the multiplicity $\gamma_{i}>0$. Then $s_{n} \in o(N(r))$ if and only if $s_{n}=\sum_{i=1}^{k}\left(1 / \alpha_{i}^{n}\right) \sum_{j=0}^{\gamma_{i}-1} c_{i j} A_{n}^{j}+t_{n}$, where

$$
t_{n}=o(1), \quad A_{n}^{j}=\left(\begin{array}{c}
n+j \\
n
\end{array}\right) \quad\left(c_{i j}=\text { constant }\right),
$$

and this result remains true with a in place of $o$.

When $r(z)$ is a polynomial, the $o$ case of the last result recently was proved by Petersen. ${ }^{5}$

We remark that the condition $r(z) \neq 0$ for $|z|=1$ is essential as is easily seen ${ }^{6}$ from the example $p(z)=1 /(1-z), r(z)=1+z e^{\imath \vartheta}-\pi<\vartheta$ $<+\pi$. If $s_{n} \in o(N(p))=o\left(C_{1}\right)$, we have $s_{n}=o(n)$, and if $s_{n} \in o(N(r))$ we have $s_{n}=o(n)$, but if $s_{n} \in o(N(q))$ we have $s_{n}=o\left(n^{2}\right)$ while $s_{n}=o\left(n^{\alpha}\right)$ is not true for any $\alpha<2$ and all $s_{n} \in o(N(q))$.

We shall apply our results to the methods of Cesàro and Riesz (discontinuous), and we shall obtain the difference between the convergence fields of these methods with index $2 k+1$ ( $k$ an integer).

1. The main theorem. Consider a sequence $\left\{p_{n}\right\}$ of complex numbers with the property

${ }^{4}$ The regularity of $p(z)$ for $|z|<1$ follows easily from $P_{n+1} / P_{n}=1+o(1)$ because of (2). Cf. Hardy [1, p. 65].

5 Petersen [5, Theorem 2.2, p. 452].

- By use of the Toeplitz theorem. 
$p_{0} \neq 0$, and there exists a number $N$ such that $\sum_{\nu=0}^{n} p_{\nu} \neq 0$ for $n \geqq N$.

Let $N_{0} \geqq 0$ be the smallest number $N$ with $\sum_{\nu=0}^{n} p_{\nu} \neq 0$ for $n \geqq N$. We define a sequence $\left\{P_{n}\right\}$ by $P_{n}=\sum_{\nu=0}^{n} p_{v}$ for $n \geqq N_{0}, P_{n}=1$ for $0 \leqq n<N_{0}{ }^{7}$ If a sequence $\left\{p_{n}\right\}$ satisfies the conditions (5) and (2), the functions $\sum p_{n} z^{n}$ and $\sum P_{n} z^{n}$ are regular for $|z|<1$ (because of (2), cf. footnote 3), and if $s_{n} \in o(N(p))$ or $s_{n} \in a(N(p))$, where $N(p)$ is the Nörlund mean (1), the series $\sum s_{n} z^{n}$ has a positive radius of convergence. ${ }^{8}$

From (5) and (2) we obtain the relations

$$
\begin{aligned}
p_{n+k}=o\left(P_{n}\right), \quad P_{n+k} / P_{n} & =1+o(1) \\
(n \rightarrow \infty, k & =0, \pm 1, \pm 2, \cdots) .
\end{aligned}
$$

LEMMA 1. If $a_{n}=a(1), b_{n}=a(1)$, then $a_{n}+b_{n}=a(1), a_{n} b_{n}=a(1)$. If $a_{n}=a(1)$ and $a_{n} \rightarrow c \neq 0$, then $a_{n}^{-1}=a(1)$.

The proof of Lemma 1 is trivial.

If a sequence $\left\{p_{n}\right\}$ satisfies the conditions (5), (2), and

$$
p_{n}=a\left(P_{n}\right) \quad(n \rightarrow \infty),
$$

then we obtain by use of Lemma 1 the relations

$$
\begin{aligned}
p_{n+k}=a\left(P_{n}\right), \quad P_{n+k} / P_{n} & =a(1) \\
& (n \rightarrow \infty, k=0, \pm 1, \pm 2, \cdots) .
\end{aligned}
$$

Lemma 2. Consider a sequence subject to the conditions (5) and (2). Given a number $\epsilon>0$, there exists a number $K_{1}$ with

$$
\left|P_{n+k} / P_{n}\right| \leqq K_{1}(1+\epsilon)^{|k|} \text { for } n \geqq 0, \quad k=0, \pm 1, \pm 2, \cdots,
$$

$K_{1}$ independent of $k\left(P_{-1}=P_{-2}=\cdots=0\right)$.

Proof. From (6) we obtain the relation $1 /(1+\epsilon) \leqq\left|P_{n+1} / P_{n}\right|$ $\leqq 1+\epsilon$ for $n \geqq n_{0}(\epsilon)$, and the estimation (9) follows by an easy consideration.

Lemma 3. Consider a sequence $\left\{p_{n}\right\}$ subject to conditions (5), (2), and (7). Given a number $\epsilon>0$, there exists a number $K_{2}$ with

(10) $\sum_{n=0}^{\infty}\left|\frac{P_{n+k}}{P_{n}}-\frac{P_{n+1+k}}{P_{n+1}}\right| \leqq K_{2}|k|(1+\epsilon)^{|k|}$ for $k=0, \pm 1, \pm 2, \cdots$,

${ }^{7}$ If we change a finite number of the numbers $P_{n}$ in (1), the convergence field $o(N(p))$ respectively $a(N(p))$ will not be altered. Therefore, in order to avoid some (formal) difficulties, we define the numbers $P_{n}$ so that $P_{n} \neq 0$ for $n \geqq 0$.

${ }^{8}$ Because of $s(z)=\sum s_{n} z^{n}=(p(z))^{-1} \sum P_{n \sigma_{n}} z^{n}, p(0) \neq 0$. Cf. Hardy $[1$, p. 65]. 
$K_{2}$ independent of $k\left(P_{-1}=P_{-2}=\cdots=0\right)$.

Proof. From

$$
\left(P_{n+k} / P_{n}-P_{n+1+k} / P_{n+1}\right)=\left(P_{n+k} / P_{n+1+k}-P_{n} / P_{n+1}\right) P_{n+1+k} / P_{n}
$$

(where $n+k+1 \geqq 0$ in case $k<0$ ) the estimation (10) follows because of (8) and (9).

Lemma 4. Consider a sequence $\left\{p_{n}\right\}$ subject to conditions (5) and (2). If $\left\{d_{n}\right\}$ is a sequence with $\sum \zeta^{n}\left|d_{n}\right|<\infty$ for some $\zeta>1$, then

$$
\sum_{v=n}^{\infty} b_{v} d_{v-n}=\left\{\begin{array}{l}
o\left(P_{n}\right) \\
P_{n}(d+o(1))\left(d=\sum d_{n}\right)
\end{array} \quad \text { if } b_{n}=\left\{\begin{array}{l}
o\left(P_{n}\right) \\
P_{n}(1+o(1)),
\end{array}\right.\right.
$$

and

$$
\sum_{n=0}^{n} b_{n} d_{n \rightarrow p}=\left\{\begin{array}{l}
o\left(P_{n}\right) \\
P_{n}(d+o(1))
\end{array} \quad \text { if } b_{n}=\left\{\begin{array}{l}
o\left(P_{n}\right) \\
P_{n}(1+o(1)) .
\end{array}\right.\right.
$$

Proof. Because of (9) (with $1+\epsilon<\zeta$ ) we have for any $m=n+c$ $(c \geqq 0)$

$$
\sum_{\nu=m}^{\infty}\left|\frac{P_{\nu}}{P_{n}}\right|\left|d_{v-n}\right| \leqq K_{1} \sum_{\nu=m}^{\infty}(1+\epsilon)^{n-n}\left|d_{\nu-n}\right| \leqq K_{1} \sum_{n=c}^{\infty} \zeta^{\nu}\left|d_{\nu}\right|,
$$

and from this estimation the relation (11) follows at once (observing relation (6)). In a similar manner we obtain the estimation (12) (notice that $\left|d_{n} / P_{n}\right| \leqq K_{1}(1+\epsilon) n\left|d_{n}\right|=o(1)$ ).

Lemma 5. Consider a sequence $\left\{p_{n}\right\}$ subject to conditions (5), (2), and (7). If $\left\{d_{n}\right\}$ is a sequence with $\sum \zeta^{n}\left|d_{n}\right|<\infty$ for some $\zeta>1$, then

$$
\sum_{n=n}^{\infty} b_{n} d_{v-n}=a\left(P_{n}\right) \quad \text { if } b_{n}=a\left(P_{n}\right),
$$

and

$$
\sum_{n=0}^{n} b_{\nu} d_{n-p}=a\left(P_{n}\right) \quad \text { if } b_{n}=a\left(P_{n}\right) .
$$

Proof. Consider a sequence $\delta_{n}=a(1)$. From

$$
\begin{array}{r}
\delta_{n+k} P_{n+k} / P_{n}-\delta_{n+k+1} P_{n+k+1} / P_{n+1} \\
=\delta_{n+k}\left(P_{n+k} / P_{n}-P_{n+k+1} / P_{n+1}\right)+P_{n+k+1} / P_{n+1}\left(\delta_{n+k}-\delta_{n+k+1}\right) \\
\left(\delta_{\nu}=0 \text { and } P_{\nu}=0 \text { in case } \nu<0\right)
\end{array}
$$

we obtain (because of (9) and (10)) the relation 


$$
\begin{aligned}
\sum_{n=0}^{\infty}\left|\delta_{n+k} \frac{P_{n+k}}{P_{n}}-\delta_{n+1+k} \frac{P_{n+1+k}}{P_{n+1}}\right| \leqq K_{8} K_{2}|k|(1+\epsilon)^{|k|} \\
+K_{1}(1+\epsilon)^{|k|} K_{4} \leqq K_{5}(|k|+1)(1+\epsilon)^{|k|}
\end{aligned}
$$

$(k=0, \pm 1, \pm 2, \cdots)$ with $K_{5}$ independent of $k$.

Putting $b_{n}=\delta_{n} P_{n}$ and $\beta_{n}=\sum_{\nu=n}^{\infty}\left(b_{\nu} / P_{n}\right) d_{n-n}$ we have

$$
\beta_{n}-\beta_{n+1}=\sum_{\nu=0}^{\infty}\left(\delta_{n+\nu} \frac{P_{n+\nu}}{P_{n}}-\delta_{n+1+\nu} \frac{P_{n+1+\nu}}{P_{n+1}}\right) d_{n}
$$

and by use of (15) we obtain

$$
\sum_{n=0}^{\infty}\left|\beta_{n}-\beta_{n+1}\right| \leqq K_{5} \sum_{n=0}^{\infty}(\nu+1)(1+\epsilon)^{\nu}\left|d_{\nu}\right|=K_{\epsilon} \quad(1+\epsilon<\zeta)
$$

which proves (13).

By use of (15) we obtain (14) in a similar manner.

LEMMA 6. Let $r(z)=\sum r_{n} z^{n}$ be convergent for $|z| \leqq \rho, \rho>1, r(0) \neq 0$ ' $r(1) \neq 0$, and define $q(z)$ by $q(z)=r(z) p(z)\left(q(z)=\sum q_{n} z^{n}, p(z)=\sum p_{n} z^{n}\right)$.

(i) If $\left\{p_{n}\right\}$ satisfies conditions (5) and (2), then $\left\{q_{n}\right\}$ satisfies (5) and (2), and the relation

$$
Q_{n} / P_{n}=r(1)+o(1)
$$

is true. (Because of (5) the sequence $\left\{Q_{n}\right\}$ may be generated by $\left\{q_{n}\right\}$ in the same way as is $\left\{P_{n}\right\}$ by $\left\{p_{n}\right\}$.)

(ii) If $\left\{p_{n}\right\}$ satisfies conditions (5), (2), and (7), then $\left\{q_{n}\right\}$ satisfies (5), (2), and (7), and the relation

$$
Q_{n} / P_{n}=a(1)
$$

is true.

Proof. Putting $Q_{n}^{*}=\sum_{v=0}^{n} q_{n}, P_{n}^{*}=\sum_{v=0}^{n} p_{\nu}\left(P_{n}^{*}=P_{n} \text { for } n \geqq N_{0}\right)^{\prime}$ we have the relation

$$
Q_{n}^{*}=\sum_{n=0}^{n} P_{\nu}^{*} r_{n-\nu}
$$

If $\left\{p_{n}\right\}$ is subject to conditions (5) and (2), we obtain from (18) by (12) the relation

$$
Q_{n}^{*} / P_{n}^{*}=r(1)+o(1) \quad(n \rightarrow \infty),
$$

and therefore $\left\{q_{n}\right\}$ satisfies the condition (5) (notice that $q_{0}=r_{0} p_{0}$ ). Considering $q_{n} / Q_{n}=q_{n} / P_{n} \cdot P_{n} / Q_{n}$ we obtain the statements of the 
lemma from the relation $q_{n}=\sum_{\nu=0}^{n} p_{\nu} r_{n-\nu}$, by (19), Lemma 4, Lemma 5, and Lemma 1.

Lемма 7. Let $\hat{p}(z)=\sum \hat{p}_{n} z^{n}$ be convergent for $|z|<1$, and define $p(z)$ by $p(z)=(1-z / \alpha)^{k} \hat{p}(z)$ with $0<|\alpha|<1$ and $k>0$ an integer.

(i) If $\left\{p_{n}\right\}$ satisfies conditions (5) and (2), then $\left\{\hat{p}_{n}\right\}$ satisfies (5) and (2), and the relation

$$
\widehat{P}_{n} / P_{n}=(1-1 / \alpha)^{-k}+o(1)
$$

is true. (For the definition of $\widehat{P}_{n} c f$. Lemma 6 (i).)

(ii) If $\left\{p_{n}\right\}$ satisfies conditions (5), (2), and (7), then $\left\{\hat{p}_{n}\right\}$ satisfies (5), (2), and (7), and the relation

$$
\widehat{P}_{n} / P_{n}=a(1)
$$

is true.

Proof. Suppose first $k=1$. Putting $P_{n}^{*}=\sum_{v=0}^{n} p_{v}\left(P_{n}^{*}=P_{n}\right.$ for $\left.n \geqq N_{0}\right)$ and $\widehat{P}_{n}^{*}=\sum_{\nu=0}^{n} \hat{p}_{\nu}$, we obtain from $\hat{p}(z)=(1-z / \alpha)^{-1} p(z)$ the relations

$$
\left.\widehat{p}_{n}=\frac{1}{\alpha^{n}} \sum_{\nu=0}^{n} p_{\nu} \alpha^{\nu}=-\sum_{\nu=n+1}^{\infty} p_{\nu} \alpha^{\nu-n} \quad \text { (because of } p(\alpha)=0\right),
$$

and

$$
\widehat{P}_{n}^{*}=\frac{1}{\alpha^{n}} \sum_{\nu=0}^{n} P_{\nu}^{*} \alpha^{\nu}=-\sum_{\nu=n+1}^{\infty} P_{\nu} \alpha^{\nu-n} \quad \text { for } n \geqq N_{0} .
$$

If $\left\{p_{n}\right\}$ is subject to conditions (5) and (2), then we obtain from (23) by (11) (putting $d_{n}=\alpha^{u+1}$ ) the relation

$$
\widehat{P}_{n}^{*} / P_{n}=(1-1 / \alpha)^{-1}+o(1),
$$

hence (because of $\hat{p}_{0}=p_{0}$ ) $\left\{\hat{p}_{n}\right\}$ satisfies the relation (5). Considering $\hat{p}_{n} / \widehat{P}_{n}=\hat{p}_{n} / P_{n} \cdot P_{n} / \widehat{P}_{n}$ we derive the statements of the lemma from (22) and (24) by Lemma 4, Lemma 5 (putting $d_{n}=\alpha^{n+1}$ ), and Lemma 1.

For $k>1$ the result follows by induction.

LEMMA 8. Consider a function $p(z)=\sum p_{n} z^{n}$, regular for $|z|<1$, and a number $\alpha$ with $0<|\alpha|<1$. Suppose that $p(z)$ has a root of multiplicity $\lambda \geqq 0$ for $z=\alpha$ (i.e. $p(z)=(1-z / \alpha)^{\lambda} h(z)$ where $h(z)=\sum h_{n} z^{n}$ is regular for $|z|<1$ and $h(\alpha) \neq 0)$, and define $q(z)$ by $q(z)=(1-z / \alpha) p(z)$.

(i) If $\left\{p_{n}\right\}$ satisfies conditions (5) and (2), then $\left\{q_{n}\right\}$ satisfies conditions (5) and (2), and $s_{n} \in o(N(q))$ if and only if 


$$
\begin{aligned}
& s_{n}=t_{n}+c \frac{A_{n}^{\lambda}}{\alpha^{n}}, \\
& \quad t_{n} \in o(N(p)),\left(A_{n}^{\lambda}=\left(\begin{array}{c}
n+\lambda \\
n
\end{array}\right), c=\text { constant }\right) .
\end{aligned}
$$

(ii) If $\left\{p_{n}\right\}$ satisfies conditions (5), (2), and (7), then $\left\{q_{n}\right\}$ satisfies conditions (5), (2), and (7), and $s_{n} \in a(N(q))$ if and only if

$$
s_{n}=t_{n}+c\left(A_{n}^{\lambda} / \alpha^{n}\right), \quad t_{n} \in a(N(p)) .
$$

Proof. Suppose that $\left\{p_{n}\right\}$ is subject to conditions (5) and (2). By Lemma 6 the sequence $\left\{q_{n}\right\}$ satisfies conditions (5) and (2). Consider a sequence $s_{n} \in o(N(q))$. The series $s(z)=\sum s_{n} z^{n}$ has a positive radius of convergence, and we define a function $t(z)=\sum t_{n} z^{n}$ by $t(z)=s(z)$ $-c(1-z / \alpha)^{-(\lambda+1)} \quad(c=$ constant $)$. Putting $q(z) s(z)=a(z)=\sum a_{n} z^{n}$ $\left(a_{n}=o\left(Q_{n}\right)\right.$ because of $s_{n} \in o(N(q))$, so that $a(z)$ is regular for $\left.|z|<1\right)$, we have

$$
\begin{aligned}
p(z) t(z) & =\frac{q(z)}{1-z / \alpha}\left(s(z)-c(1-z / \alpha)^{-(\lambda+1)}\right)=\frac{1}{1-z / \alpha}(a(z)-c h(z)) \\
& =\frac{1}{1-z / \alpha} \sum b_{n} z^{n} \quad\left(b_{n}=a_{n}-c h_{n}\right) .
\end{aligned}
$$

If we put $c=a(\alpha) / h(\alpha)$, we have

$$
\left.p(z) t(z)=-\sum_{n=0}^{\infty} z^{n} \sum_{n=n+1}^{\infty} b_{\nu} \alpha^{\eta-n} \quad \text { (because of } \sum b_{n} \alpha^{n}=0\right) .
$$

From (16) and $a_{n}=o\left(Q_{n}\right)$ we obtain $a_{n}=o\left(P_{n}\right)$, and by use of Lemma 7 we have

$$
h_{n}=o\left(P_{n}\right) \text {, }
$$

so that $b_{n}=o\left(P_{n}\right)$, and from this estimation we get

$$
\sum_{\nu=n+1}^{\infty} b_{\nu} \alpha^{\nu-n}=o\left(P_{n}\right)
$$

by Lemma 4. As is seen from (27), the estimation (29) means that $t_{n} \in o(N(p))$.

It remains to show that any sequence (25) is contained in $o(N(q))$. If $t_{n} \in o(N(p))$ we have $p(z) t(z)=b(z)=\sum b_{n} z^{n}$ with $b_{n}=o\left(P_{n}\right)$. Putting $q(z) t(z)=a(z)=\sum a_{n} z^{n}=(1-z / \alpha) b(z)$, we obtain $a_{n}=o\left(P_{n}\right)$ (by 
use of (12)), and because of $Q_{n}=P_{n}(1-1 / \alpha+o(1)$ ) (by Lemma 6) we have $a_{n}=o\left(Q_{n}\right)$ so that $t_{n} \in o(N(q))$.

Finally we have $A_{n}^{\lambda} / \alpha^{n} \in o\left(N\left(q_{n}\right)\right)$ because of $q(z) \cdot \sum_{n=0}^{\infty}\left(A_{n}^{\lambda} / \alpha^{n}\right) z^{n}$ $=h(z),(28)$, and (16).

Writing $a$ instead of $o$, the proof of (ii) runs in exactly the same lines as the proof of (i).

LEMMA 9. Consider a function $p(z)=\sum p_{n} z^{n}$, regular for $|z|<1$, and a function $r(z)=\sum r_{n} z^{n}$, regular for $|z| \leqq 1$ with $r(z) \neq 0$ for $|z| \leqq 1$. Define $q(z)$ by $q(z)=r(z) p(z)$.

(i) If $\left\{p_{n}\right\}$ satisfies conditions (5) and (2), then $\left\{q_{n}\right\}$ satisfies conditions (5) and (2), and we have $o(N(p))=o(N(q))$.

(ii) If $\left\{p_{n}\right\}$ satisfies conditions (5), (2), and (7), then $\left\{q_{n}\right\}$ satisfies conditions (5), (2), and (7), and we have $a(N(p))=a(N(q))$.

Proof. Suppose that $\left\{p_{n}\right\}$ is subject to conditions (5) and (2). By Lemma 6 the sequence $\left\{q_{n}\right\}$ satisfies conditions (5) and (2). Consider a sequence $s_{n} \in o(N(p))$. Putting

$$
p(z) s(z)=a(z)=\sum a_{n} z^{n}\left(s(z)=\sum s_{n} z^{n}\right),
$$

we have $a_{n}=o\left(P_{n}\right)$. Putting $q(z) s(z)=b(z)=\sum b_{n} z^{n}=r(z) a(z)$ we have the relation

$$
b_{n}=\sum_{v=0}^{n} a_{v} r_{n-v}
$$

Using Lemma 4, we derive from (30) the relation $b_{n}=o\left(P_{n}\right)$, and by Lemma 6 we obtain $b_{n}=o\left(Q_{n}\right)$. Hence we have $s_{n} \in o(N(q))$ and therefore $o(N(p)) \subseteq o(N(q))$. From $p(z)=r(z)^{-1} q(z)$ where $r(z)^{-1}$ satisfies the same conditions as does $r(z)$, we obtain the relation $o(N(q))$ $\subseteq o(N(p))$, and this yields the result desired.

The proof of (ii) runs in exactly the same lines as the preceeding proof of (i).

The following theorem is obtained by a combination of Lemma 8 and Lemma 9.

THEOREM 1. Consider a function $r(z)=\sum r_{n} z^{n}$, regular for $|z| \leqq 1$, $r(z) \neq 0$ for $z=0$ and $|z|=1$, and having inside the unit circle the roots $\alpha_{1} \cdots \alpha_{k}$ with the multiplicities $\gamma_{1} \cdots \gamma_{k}$

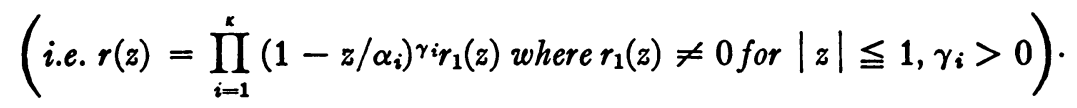

Let $p(z)=\sum p_{n} z^{n}$ be a function regular for $|z|<1$ and suppose 
that $a_{i}$ (i.e. the ith root of $r(z)$ ) is a root of $p(z)$ with multiplicity $\lambda_{i} \geqq 0(i=1,2, \cdots, k)$. Define $q(z)$ by $q(z)=r(z) p(z)$.

(i) If $\left\{p_{n}\right\}$ satisfies conditions (5) and (2), then $s_{n} \in o(N(q))$ if and only if

$$
\begin{aligned}
s_{n}=t_{n}+ & \sum_{i=1}^{\kappa} \frac{1}{\alpha_{i}^{n}} \sum_{j=\lambda_{i}}^{\lambda_{i}+\gamma_{i}-1} c_{i j} A_{n}^{j}, \\
t_{n} & \in o(N(p)),\left(A_{n}^{j}=\left(\begin{array}{c}
n+j \\
n
\end{array}\right), c_{i j}=\text { constant }\right) .
\end{aligned}
$$

(ii) If $\left\{p_{n}\right\}$ satisfies conditions (5), (2), and (7), then $s_{n} \in a(N(q))$ if and only if (31) holds with $t_{n} \in a(N(p))$.

In case (i) the sequence $\left\{q_{n}\right\}$ satisfies conditions (5) and (2), and in case (ii) the sequence $\left\{q_{n}\right\}$ satisfies (5), (2), and (7).

Proof. Considering the functions

$$
\begin{aligned}
& \left(1-z / \alpha_{1}\right) p(z),\left(1-z / \alpha_{1}\right)^{2} p(z), \cdots,\left(1-z / \alpha_{1}\right)^{\gamma_{1}} p(z), \\
& \left(1-z / \alpha_{2}\right)\left(1-z / \alpha_{1}\right)^{\gamma_{1}} p(z), \cdots, \prod_{i=1}^{\kappa}\left(1-z / \alpha_{i}\right)^{\gamma_{i}} p(z)=p^{*}(z),
\end{aligned}
$$

we obtain by a repeated application of Lemma 8 the result that the convergence field of $p^{*}(z)$ is given by (31) (respectively by (31) with $t_{n} \in a(N(p))$ in case (ii)). By Lemma 9 the convergence fields of the Nörlund means belonging to $p^{*}(z)$ and $r_{1}(z) p^{*}(z)=q(z)$ are not different.

Finally we shall show that the theorems stated in the introduction of this paper are special cases of Theorem 1 . These theorems deal with regular (absolutely regular) Nörlund means while the weaker conditions (5) and (2) ((5), (2), and (7)) imply that the $N(p)$-transformation of any sequence $\left\{s_{n}\right\}$ with $s_{n}=0$ for all large $n$ tends to zero (tends to zero and is absolutely convergent).

Given a regular (absolutely regular) Nörlund mean ${ }^{9} N(p)$ and a sequence $\left\{r_{n}\right\}$ such that $r(z)=\sum r_{n} z^{n}$ is convergent for $|z| \leqq \zeta, \zeta>1$, $r(0) \neq 0$ and $r(1) \neq 0$, then by $q(z)=r(z) p(z)$ a regular (absolutely regular) Nörlund mean $N(q)$ is defined. In fact, suppose that $s_{n}=o(1)$ $\left(s_{n}=o(1)\right.$ and $\left.s_{n}=a(1)\right) \cdot{ }^{10}$ Putting

$$
\sigma_{n}=\left(1 / P_{n}\right) \sum_{v=0}^{n} p_{n \rightarrow>} s_{\nu}
$$

- And $\left\{p_{n}\right\}$ subject to condition (5).

${ }^{10}$ Obviously it is sufficient to consider only sequences tending to zero. 
we have

$$
\sum_{\nu=0}^{n} q_{n-\nu} s_{\nu}=\sum_{\nu=0}^{n} r_{n-\nu} P_{\nu} \sigma_{\nu}=o\left(P_{n}\right)\left(=o\left(P_{n}\right) \text { and }=a\left(P_{n}\right)\right)
$$

by Lemma 4 (Lemma 4 and Lemma 5) because of $\sigma_{n}=o(1)\left(\sigma_{n}=o(1)\right.$ and $\left.\sigma_{n}=a(1)\right)$ and from (16) ((16) and (17)) we obtain the relation $\sum_{\nu=0}^{n} q_{n \rightarrow \nu} S_{\nu}=o\left(Q_{n}\right)\left(=o\left(Q_{n}\right)\right.$ and $\left.=a\left(Q_{n}\right)\right)$ q.e.d.

If we put $p(z)=1$, the last theorem of the introduction follows at once from Theorem 1, and combining this result again with Theorem 1 we obtain the first and the second theorem of the introduction (the second term in (31) (with $\lambda_{i}=0$ ) plus all sequences tending to zero represents all sequences belonging to $o(N(r))$, similarly in the absolute case).

2. An application. As an application of Theorem 1 we shall investigate the convergence fields of the Cesàro means $C_{k}=N(p)$ with

$$
p_{n}=\left(\begin{array}{c}
n+k-1 \\
n
\end{array}\right)
$$

and the discontinuous Riesz means $R_{n}^{k}=N(q)$ with $q_{n}=(n+1)^{k}-n^{k}$ $(k>0)$. We shall assume that $k$ is an integer. The sequences $\left\{p_{n}\right\}$ and $\left\{q_{n}\right\}$ thus defined satisfy conditions (2), (5), and (7), and $C_{k}$ is connected with the function $p(z)=1 /(1-z)^{k}$ while $R_{n}^{k}$ is connected with the function

$$
q(z)=(1-z) \sum_{n=0}^{\infty}(n+1)^{k} z^{n}=(1-z)\left(\frac{d}{d z} z\right)^{k} \frac{1}{1-z}=\frac{P_{k-1}(z)}{(1-z)^{k}}
$$

$P_{k-1}(z)$ being a polynomial of degree $k-1$ (this follows easily by induction from $\left.P_{0}=1, P_{1}=1+z, P_{2}=1+4 z+z^{2}\right)$.

From (32) we obtain the relation $q(z)=P_{k-1}(z) p(z)$, and therefore, in order to apply Theorem 1 , we have to investigate the distribution of the roots of $P_{k-1}(z) .{ }^{11}$

LEMma 10. The polynomials $P_{k}(z)(k=0,1, \cdots)$ are reciprocals, i.e. $P_{k}(z)=z^{k} P_{k}(1 / z)$.

Proof. Starting with $P_{0}(z)=1$, we proceed by induction. Assuming that $P_{k-1}(z)=z^{k-1} P_{k-1}(1 / z)(k \geqq 1)$, we have for $k \geqq 1$ the relation ${ }^{12}$

" Some properties of the function $\sum(n+1)^{k} z^{n}$ (including the distribution of the roots) have been investigated by Lawden [3]. Here we shall give a short proof of those properties of the roots of $P_{k-1}(z)$ we need for our purposes.

12 Because of the (formal) relation $\left.(d / d \zeta) f(\zeta)\right|_{\zeta-1 / \mathrm{a}}=-\left.x^{2}(d / d x) f(1 / x)\right|_{x-\mathrm{a}}$. 


$$
\begin{aligned}
P_{k}\left(\frac{1}{z}\right) & =\left.\left(1-\frac{1}{z}\right)^{k+2} \frac{d}{d \zeta} \frac{\zeta P_{k-1}(\zeta)}{(1-\zeta)^{k+1}}\right|_{\zeta=1 / z} \\
& =-z^{2}\left(1-\frac{1}{z}\right)^{k+2} \frac{d}{d z} \frac{(1 / z) P_{k-1}(1 / z)}{(1-1 / z)^{k+1}} \\
& =-z^{2}\left(1-\frac{1}{z}\right)^{k+2} \frac{d}{d z} \frac{z P_{k-1}(z)}{(z-1)^{k+1}} \\
& =-z^{2}\left(1-\frac{1}{z}\right)^{k+2}(-1)^{k+1} \frac{P_{k}(z)}{(1-z)^{k+2}}=\frac{1}{z^{k}} P_{k}(z)
\end{aligned}
$$

LEMMA 11. All roots of $P_{k}(z)(k \geqq 0)$ are located on the negative real axis. Exactly $k$ different roots of $P_{2 k}(z)$ and $P_{2 k+1}(z)$ are contained in any of the intervals $-\infty<z<-1,-1<z<0$, and we have $P_{2 k}(-1) \neq 0$, $P_{2 k+1}(-1)=0$.

Proof. Putting $f_{k}(z)=P_{k}(z) /(1-z)^{k+2}$ we have $f_{k+1}(z)=\left(z f_{k}(z)\right)^{\prime}$ $(z \neq 1)$, and by integration the formula

$$
\int_{a}^{b} f_{k+1}(x) d x=b f_{k}(b)-a f_{k}(a) \quad(x \text { real, } a<b<+1, k \geqq 0) .
$$

From (33) we derive at once the following properties of $P_{k}(z)$ :

(i) Given two real numbers $\alpha$ and $\beta$ with $\alpha<\beta<0$ and $P_{k}(\alpha)=P_{k}(\beta)$ $=0$, there exists a real number $\gamma$ with $\alpha<\gamma<\beta$ such that $P_{k+1}(\gamma)=0$ (put $a=\alpha, b=\beta$ in (33)).

(ii) Given a real number $\alpha<0$ with $P_{k}(\alpha)=0$, there exists a real number $\gamma$ with $\alpha<\gamma<0$ such that $P_{k+1}(\gamma)=0$ (put $b=0, a=\alpha$ in (33)).

Starting with $P_{1}(z)=1+z$ and proceeding by induction, we obtain the result desired by an easy consideration from (i), (ii), and Lemma ${ }^{13}$ 10 (observe that $P_{k}$ has at most $k$ different roots).

Using Lemma 11, we derive from Theorem 1 the following

THEOREM 2. A sequence $\left\{s_{n}\right\}$ is an element of $o\left(R_{n}^{2 k+1}\right)(k \geqq 0$ an integer) $\left(a\left(R_{n}^{2 k+1}\right)\right)$ if and only if

$$
s_{n}=t_{n}+\sum_{i=1}^{k} \frac{c_{i}}{\alpha_{i}^{n}}, \quad t_{n} \in o\left(C_{2 k+1}\right)\left(t_{n} \in a\left(C_{2 k+1}\right)\right)
$$

where $c_{i}(i=1,2, \cdots, k)$ is constant and the numbers $\alpha_{i}$ are the roots of $P_{2 k}(z)$ located inside the unit circle.

It may be of some interest to have an estimation for the modulus of the smallest root of $P_{k}(z)$.

${ }^{13}$ By Lemma 10 we have $P_{2 k+1}(-1)=0$ and $P_{k}\left(1 / z_{0}\right)=0$ if $P_{k}\left(z_{0}\right)=0$. 
LEMma 12. Let $\alpha_{0}(k)$ denote the root with smallest modulus of $P_{k}(z)$. Then we have the estimation

$$
\begin{aligned}
\frac{1+o(1)}{2^{k+1}} \leqq\left|\alpha_{0}(k)\right| \leqq k \frac{1+o(1)}{2^{k+1}} & \\
& \left(\text { with } 1+o(1)=\frac{1}{1-(k+2) / 2^{k+1}}\right) .
\end{aligned}
$$

Proof. From (32) we have $P_{k}(0)=1$ and therefore

$$
P_{k}(z)=\prod_{i=1}^{k}\left(z-\alpha_{i}\right)
$$

( $k \geqq 1$, observe Lemma 10 and $a_{0}=a_{n}=1$ (because of Lemma 11)). Considering the logarithmic derivative of this identity we obtain

$$
P_{k}^{\prime}(z) / P_{k}(z)=-\sum_{n=0}^{\infty} z^{n} \sum_{i=1}^{k} 1 / \alpha_{j}^{n+1}|z| \text { being small. }
$$

From $P_{k}^{\prime}(0) / P_{k}(0)=\sum_{l=1}^{k} 1 /\left|\alpha_{i}\right|, 1 /\left|\alpha_{0}(k)\right| \leqq \sum_{i=1}^{k} 1 /\left|\alpha_{i}\right| \leqq k /\left|\alpha_{0}(k)\right|$ and $P_{k}^{\prime}(0)=2^{k+1}-(k+2)$ (this relation follows by an easy calculation from (32)) we obtain (34).

Finally we consider the Nörlund mean $N(\hat{p})$ defined by $\hat{p}_{0}=k^{k}$, $\hat{p}_{n}=(n+k)^{k}-(n-1+k)^{k}, n \geqq 1 \quad(k>0$ an integer $)$. We obtain this mean from $R_{n}^{k}$ omitting the first $(k-1)$ elements of the sequence $\left\{p_{n}\right\} .{ }^{14}$ Similarly to $(32)$ we have for $p(z)$ the relation

$$
\begin{aligned}
p(z) & =(1-z) \sum_{n=0}^{\infty}(n+k){ }^{k} z^{n}=(1-z) \frac{1}{z^{k-1}}\left(\frac{d}{d z} z\right)^{k} \frac{z^{k-1}}{1-z} \\
& =\frac{Q_{k}(z)}{(1-z)^{k}} .
\end{aligned}
$$

We have $Q_{1}(z)=1, Q_{2}(z)=z^{2}-3 z+4, Q_{3}(z)=-8 z^{3}+31 z^{2}-44 z+27$, $Q_{4}(z)=81 z^{4}-389 z^{8}+731 z^{2}-655 z+256, \quad Q_{5}(z)=-1024 z^{5}+5901 z^{4}$ $-13934 z^{3}+17026 z^{2}-10974 z+3125$.

By numerical calculation we obtain the following roots of smallest modulus

$$
\begin{array}{lll}
Q_{2}: z_{0}=1.5+i 1.32 \cdots, \quad\left(=3 / 2+i 7^{1 / 2} / 2\right), & \left|z_{0}\right|^{2}=4, \\
Q_{3}: z_{0}=0.9710+i 0.8961+\epsilon, & |\epsilon|<3.10^{-4}, & \left|z_{0}\right|^{2}=1.74 \cdots, \\
Q_{4}: z_{0}=0.80299+i 0.71437+\epsilon, & |\epsilon|<10^{-5}, & \left|z_{0}\right|^{2}=1.155 \cdots, \\
Q_{5}: z_{0}=0.72034+i 0.60896+\epsilon, & |\epsilon|<5.10^{-5}, & \left|z_{0}\right|^{2}=0.89 \cdots,
\end{array}
$$

14 A Nörlund mean of this kind has been considered by Riesz [6]. 
From Theorem 1 we see that the means $N(\hat{p})$ are equivalent with $C_{k}$ for $k=1,2,3,4$, but not for $k=5$ (and probably, not for any $k \geqq 5$ ).

\section{BibLIOGRAPHY}

1. G. H. Hardy, Divergent series, Oxford, 1949.

2. K. Knopp and G. G. Lorentz, Beiträge zur absoluten Limitierung, Archiv der Mathematik vol. 2 (1949) pp. 10-16.

3. D. F. Lawden, The function $\sum n^{r} z^{n}$ and associated polynomials, Proc. Cambridge Philos. Soc. vol. 47 (1951) pp. 309-314.

4. F. M. Mears, Absolute regularity and the Nörlund mean, Ann. of Math. vol. 38 (1937) pp. 594-601.

5. G. M. Petersen, $A$ note on divergent series, Canadian Journal of Mathematics vol. 4 (1952) pp. 445-454.

6. M. Riesz, Sur l'equivalence de certaines methodes de sommation, Proc. London Math. Soc. vol. 22 (1924) pp. 412-419.

UNIVERSITY OF GIESSEN

\section{REARRANGEMENTS OF SERIES}

H. M. SENGUPTA

1. Introduction. Professor R. P. Agnew [1] and the author [2] have considered the metric space $E$ of points $x \equiv\left(x_{1}, x_{2}, x_{3}, \cdots\right)$ where the complex $\left(x_{1}, x_{2}, x_{3}, \cdots\right)$ is a permutation of the positive integers and the distance between two points $x$ and $y$ is defined by

$$
d(x, y)=\sum_{n=1}^{\infty} \frac{1}{2^{n}} \frac{\left|x_{n}-y_{n}\right|}{1+\left|x_{n}-y_{n}\right|} .
$$

Starting with a given conditionally convergent series $\sum_{n=1}^{\infty} c_{n}$ of real terms, we associate it with the point $(1,2,3, \cdots)$ of the space $E$. Following Professor Agnew, we sometimes write the series $\sum c_{n}$ in the form $\sum c(n)$. With a given rearrangement of this series, say $c\left(n_{1}\right)+c\left(n_{2}\right)+c\left(n_{3}\right)+\cdots$, we associate in a unique manner the point $z=\left(n_{1}, n_{2}, n_{3}, \cdots\right)$ of the space $E$. For instance the rearranged series $c_{2}+c_{1}+c_{4}+c_{3}+\cdots$ is associated with the point $(2,1,4,3, \cdots)$ of $E$. Conversely, with a given point $z=\left(n_{1}, n_{2}, \cdots\right)$ of $E$ we associate the rearrangement $c\left(n_{1}\right)+c\left(n_{2}\right)+\cdots$ of the given series, and if the rearrangement converges to $\alpha$ we shall say that the series which corresponds to the point $z$ converges to $\alpha$.

Received by the editors April 18, 1955 and, in revised form, May 16, 1955. 